\title{
COMPACT UNIFORM FIELD ELECTRODE PROFILES
}

\author{
Gerard J. ERNST \\ Department of Applied Physics, Twente University of Technology, Enschede, The Netherlands
}

Received 18 April 1983

\begin{abstract}
By modification of the conformal transformation used by Chang in determining uniform field electrode profiles, a large improvement can be achieved in the uniformity of the electric field strength distribution over the surface of the electrodes. When such electrodes are used in a TEA laser system, smaller electrodes can be used for the same gas discharge width.
\end{abstract}

\section{Introduction}

In order to obtain high output powers from transversely excited pulsed lasers it is important to have a very uniform energy loading of the active gas medium. That is why there is a need for specially contoured electrodes which produce a very uniform field strength over a certain amount of surface. A number of authors have given solutions to this problem. Up to now, Rogowski profiles [1] are most commonly used, but have the serious disadvantage that three smooth segments have to be joint together in an undefined and rough manner. The best profiles are made by using the analytic formulas derived by Chang [2]. For a realistic Chang profile, however, the width of a TEA laser electrode, required to produce a square discharge, amounts to approximately 3.5 times the discharge width [3]. For some applications, however, it is desirable to have a smaller electrode-to-discharge width ratio. This is, for instance, the case for large aperture $\mathrm{CO}_{2}$ or $\mathrm{CO}$ lasers, where the distance of the UV source from the electrode centre is an important measure, or for large aperture UV lasers, where the electrode inductance contributes significantly to the total circuit inductance. Compacting the electrodes in the way Chang describes in his paper is undesirable because that leads to a decrease in the field strength uniformity at the electrode surface. That is why we looked for compacting in a different way.

\section{The conformal transformation}

We start with the same conformal transformation as used by Chang:

$\zeta=w+k(w) \sinh w$,

where $\zeta=x+\mathrm{i} y$ and $w=u+\mathrm{i} v$, with $x$ and $y$ being the space coordinates and $u$ and $v$ being the flux and potential functions, respectively. However, instead of $k$ being a constant, we assume $k$ to be a function of $w$. For every value of $v(|v|<\pi)$ the profile of the corresponding equipotential surface is given by

$x=u+\operatorname{Re}(k) \cos v \sinh u-\operatorname{Im}(k) \sin v \cosh u$,

$y=v+\operatorname{Re}(k) \sin v \cosh u+\operatorname{Im}(k) \cos v \sinh u$,

where $u$ is the running variable.

Because the profile has to be sy snmetric with respect to the $y$ axis and because the $+v$ and $-v$ equipotentials have to be mirror images with respect to the $x$ axis, the real part of $k$, designated by $\operatorname{Re}(k)$, has to be an even function with respect to $u$ as well as $v$, whereas the imaginary part of $k$, designated by $\operatorname{Im}(k)$, has to be an odd function with respect to $u$ as well as $v$. Those conditions are fulfilled when $k$ is an even function of $w$.

As can be seen from relations (2) and (3), the uniform field electrode (UFE) profile is not uniquely determined. For the case that $k$ is a real constant, $k_{0}$, two independent variables, $k_{0}$ and $v$, can be chosen, both of which determine the form of the profile as well as the electric field strength distribution. To find 
the optimum UFE profile, an expression is needed for the electric field strength:

$E^{-2}=|\mathrm{d} \zeta / \mathrm{d} w|^{2}=|1+k \cosh w+(\mathrm{d} k / \mathrm{d} w) \sinh w|^{2}$.

When the electric field strength is expressed as a power series expansion in $u$,

$E=E_{0}(k, v)+E_{2}(k, v) u^{2}+E_{4}(k, v) u^{4}+\ldots$,

the optimum profile can be found by requiring the lower coefficients (except, of course, for the first one, $E_{0}$ ) to vanish, or, if this is impossible, to be maximum. In the case of $k$ being a constant $k_{0}$, the second coefficient has to be zero or

$-\left[f(0) f^{(2)}(0)-g^{(1)^{2}}(0)\right] / f^{3}(0)=0$,

where

$f(u)=1+k_{0} \cos v \cosh u$,

$g(u)=k_{0} \sin v \sinh u$,

and where the exponent between brackets denotes the number of differentiations with respect to $u$.

From (6) the following condition follows:

$v=\arccos \left(-k_{0}\right)$,

as already has been derived by Chang.

We shall now treat two specific cases of improved UFE profiles. In the first case a fourth-power function in $w$ will be chosen for $k$ and in the second case $k$ will be an eighth-power function in $w$.

(A) $k$ is a fourth-power function in $w$.

As already mentioned, improvements of UFE profiles can be expected if $k$ is allowed to be some even function of $w$. Although, in principle, many types of functions can be tried, the easiest way is probably to take some terms of a power series expansion of $k$ around $w=0$.

In this section we will consider the case where $k$ has the following form:

$k=k_{0}+\frac{1}{2} k_{2} w^{2}+\frac{1}{24} k_{4} w^{4}$.

For the coordinates equations (2) and (3) hold where

$\operatorname{Re}(k)=k_{0}+\frac{1}{2} k_{2}\left(u^{2}-v^{2}\right)+\frac{1}{24} k_{4}\left(u^{4}+v^{4}-6 u^{2} v^{2}\right)$,

$\operatorname{Im}(k)=k_{2} u v+\frac{1}{6} k_{4}\left(u^{3} v-u v^{3}\right)$.

Now we have four free parameters, $k_{0}, k_{2}, k_{4}$, and $v$, to optimize the profile. This means that it is not only possible to require the coefficient $E_{2}$, but also to require the coefficients $E_{4}$ and $E_{6}$ from eq. (5) to vanish. So equation (6) must be satisfied together with the following two equations:

$$
\begin{aligned}
- & {\left[f(0) f^{(4)}(0)+3 f^{(2)^{2}}(0)+4 g^{(1)}(0) g^{(3)}(0)\right] / f^{3}(0)=0, } \\
- & {\left[f(0) f^{(6)}(0)+15 f^{(2)}(0) f^{(4)}(0)\right.} \\
& \left.+6 g^{(1)}(0) g^{(5)}(0)+10 g^{(3)^{2}}(0)\right] / f^{3}(0)=0,
\end{aligned}
$$

where

$f(u)=1+\operatorname{Re}(k) \cosh u \cos v-\operatorname{Im}(k) \sinh u \sin v$

$+\operatorname{Re}^{(1)}(k) \sinh u \cos v-\operatorname{Im}^{(1)}(k) \cosh u \sin v$,

$g(u)=\operatorname{Re}(k) \sinh u \sin v+\operatorname{Im}(k) \cosh u \cos v$

$+\operatorname{Re}^{(1)}(k) \cosh u \sin v+\operatorname{Im}^{(1)}(k) \sinh u \cos v$,

and where the exponent between brackets denotes the number of differentiations with respect to $u$ again.

The computer calculations show a rather capricious behaviour of the coefficient $E_{6}$ as a function of $v$ for $E_{2}$ and $E_{4}$ both zero. This behaviour is shown in fig. 1 for a $k_{0}$ value of 0.02 . The dotted part of the curve denotes negative values of $E_{6}$. Two zero points are found in this case, one for $v=1.621403$ and one for $v=3.036043$, the latter one being unimportant.

Figs. 2 and 3 show the results of our computer calculations when all three coefficients $E_{2}, E_{4}$, and $E_{6}$ equal zero. In order to make the use of this type of profile convenient to everyone, the optimum

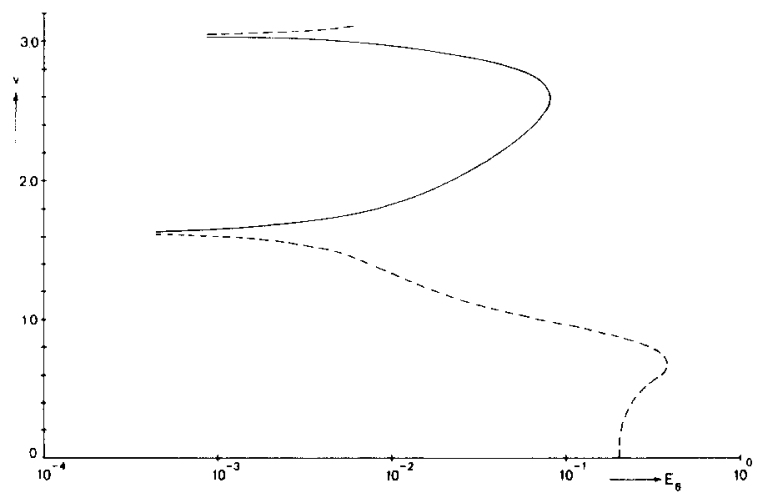

Fig. 1. The coefficient $E_{6}$ as a function of $v$ for $E_{2}$ and $E_{4}$ both zero. $k$ is a fourth-power function of $w$ and the $k_{0}$ value is 0.02 . The dotted part in the figure denotes negative values. 


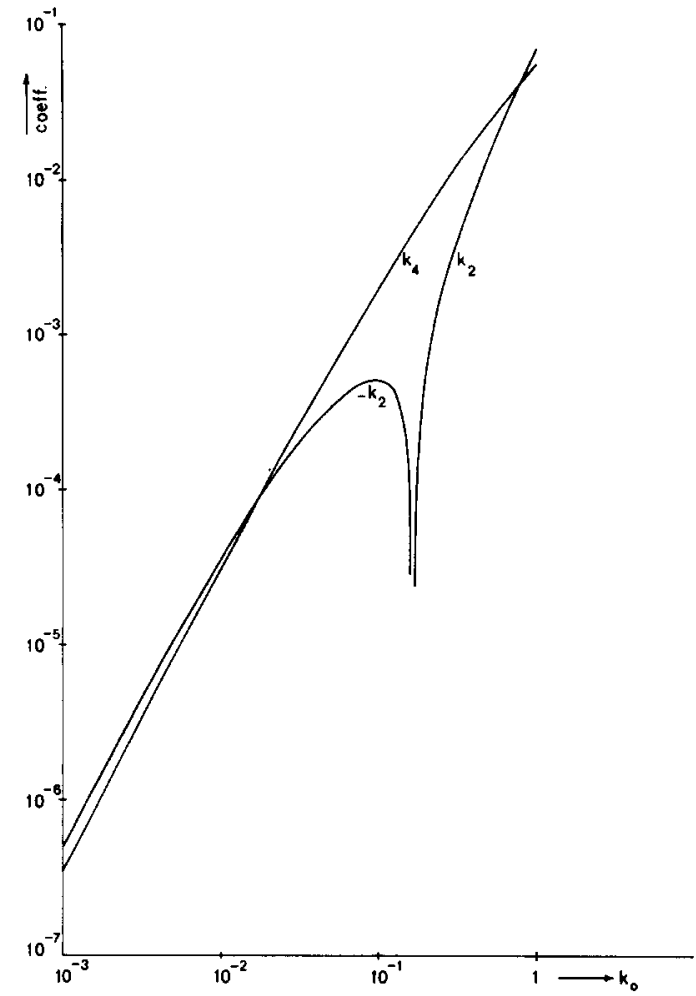

Fig. 2. The optimized values of $k_{2}$ and $k_{4}$ as a function of $k_{0}$. $k$ is a fourth-power function of $w$.

values of $k_{2}, k_{4}$, and $v$ are given in tabular form in table 1 for a range of $k_{0}$-values.

(B) $k$ is an eighth-power function in $w$.

We now consider the case where $k$ has the follow-

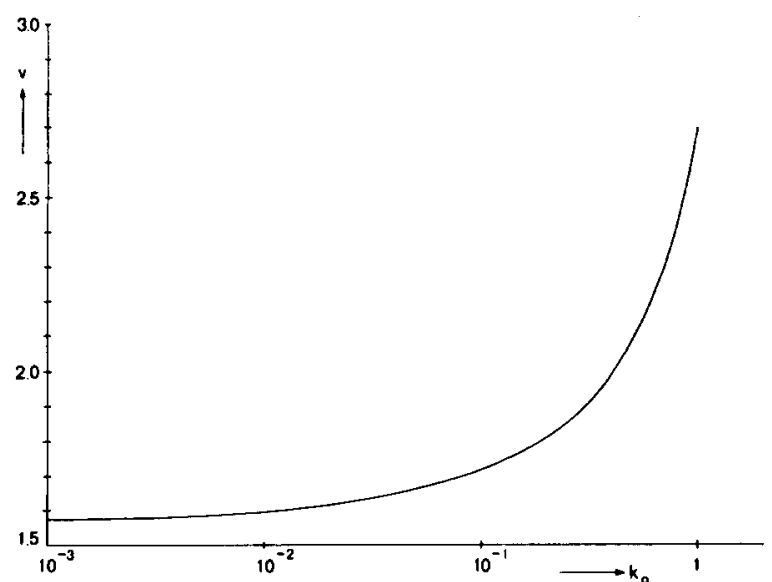

Fig. 3. The optimized value of $v$ as a function of $k_{0} \cdot k$ is a fourth-power function of $w$.
Table 1

$k=k_{0}+\frac{1}{2} k_{2} w^{2}+\frac{1}{24} k_{4} w^{4}$

\begin{tabular}{llll}
\hline$k_{0}$ & \multicolumn{1}{l}{$k_{2}$} & $k_{4}$ & $v$ \\
\hline 0.001 & $-0.4894729 \mathrm{E}-6$ & $0.3526636 \mathrm{E}-6$ & 1.574247 \\
0.0015 & $-0.1078868 \mathrm{E}-5$ & $0.7874861 \mathrm{E}-6$ & 1.575911 \\
0.002 & $-0.1880627 \mathrm{E}-5$ & $0.1389751 \mathrm{E}-5$ & 1.577540 \\
0.003 & $-0.4073689 \mathrm{E}-5$ & $0.3083106 \mathrm{E}-5$ & 1.580695 \\
0.005 & $-0.1052878 \mathrm{E}-4$ & $0.8340576 \mathrm{E}-5$ & 1.586644 \\
0.007 & $-0.1928212 \mathrm{E}-4$ & $0.1595262 \mathrm{E}-4$ & 1.592177 \\
0.01 & $-0.3577091 \mathrm{E}-4$ & $0.3148105 \mathrm{E}-4$ & 1.599834 \\
0.015 & $-0.6956990 \mathrm{E}-4$ & $0.6741250 \mathrm{E}-4$ & 1.611253 \\
0.02 & $-0.1082389 \mathrm{E}-3$ & $0.1147659 \mathrm{E}-3$ & 1.621403 \\
0.03 & $-0.1912708 \mathrm{E}-3$ & $0.2399266 \mathrm{E}-3$ & 1.639043 \\
0.05 & $-0.3465792 \mathrm{E}-3$ & $0.5945713 \mathrm{E}-3$ & 1.667832 \\
0.07 & $-0.4561044 \mathrm{E}-3$ & $0.1066652 \mathrm{E}-2$ & 1.691792 \\
0.1 & $-0.5005615 \mathrm{E}-3$ & $0.1958405 \mathrm{E}-2$ & 1.723087 \\
0.15 & $-0.2169977 \mathrm{E}-3$ & $0.3842812 \mathrm{E}-2$ & 1.769531 \\
0.2 & $0.5203643 \mathrm{E}-3$ & $0.6117696 \mathrm{E}-2$ & 1.813299 \\
0.3 & $0.3302783 \mathrm{E}-2$ & $0.1148132 \mathrm{E}-1$ & 1.900163 \\
0.5 & $0.1374477 \mathrm{E}-1$ & $0.2383356 \mathrm{E}-1$ & 2.085975 \\
0.7 & $0.3039475 \mathrm{E}-1$ & $0.3665473 \mathrm{E}-1$ & 2.297876 \\
1.0 & $0.6755833 \mathrm{E}-1$ & $0.5572676 \mathrm{E}-1$ & 2.692438 \\
\hline
\end{tabular}

ing form:

$k=k_{0}+\frac{1}{2} k_{2} w^{2}+\frac{1}{24} k_{4} w^{4}+\frac{1}{720} k_{6} w^{6}+\frac{1}{40320} k_{8} w^{8}$.

For the coordinates equations (2) and (3) hold again where now

$$
\begin{aligned}
& \operatorname{Re}(k)=k_{0}+\frac{1}{2} k_{2}\left(u^{2}-v^{2}\right)+\frac{1}{24} k_{4}\left(u^{4}+v^{4}-6 u^{2} v^{2}\right) \\
& \quad+\frac{1}{720} k_{6}\left(u^{6}-v^{6}-15 u^{4} v^{2}+15 u^{2} v^{4}\right) \\
& \quad+\frac{1}{40320} k_{8}\left(u^{8}+v^{8}-28 u^{6} v^{2}-28 u^{2} v^{6}+70 u^{4} v^{4}\right) \\
& \operatorname{Im}(k)=k_{2} u v+\frac{1}{6} k_{4}\left(u^{3} v-u v^{3}\right) \\
& \quad+\frac{1}{360} k_{6}\left(3 u^{5} v-10 u^{3} v^{3}+3 u v^{5}\right) \\
& \quad+\frac{1}{5040} k_{8}\left(u^{7} v-7 u^{5} v^{3}+7 u^{3} v^{5}-u v^{7}\right) .
\end{aligned}
$$

Six parameters, $k_{0}, k_{2}, k_{4}, k_{6}, k_{8}$, and $v$, are free to optimize the profile. This means that it is now possible to require all coefficients, $E_{2}, E_{4}, E_{6}, E_{8}$, and $E_{10}$ from eq. (5) to vanish. So equations (6), (13) and (14) must be satisfied together with the following two equations:

$$
\begin{aligned}
& -\left[f(0) f^{(8)}(0)+28 f^{(2)}(0) f^{(6)}(0)+35 f^{(4)^{2}}(0)\right. \\
& \left.+8 g^{(1)}(0) g^{(7)}(0)+56 g^{(3)}(0) g^{(5)}(0)\right] / f^{3}(0)=0,
\end{aligned}
$$




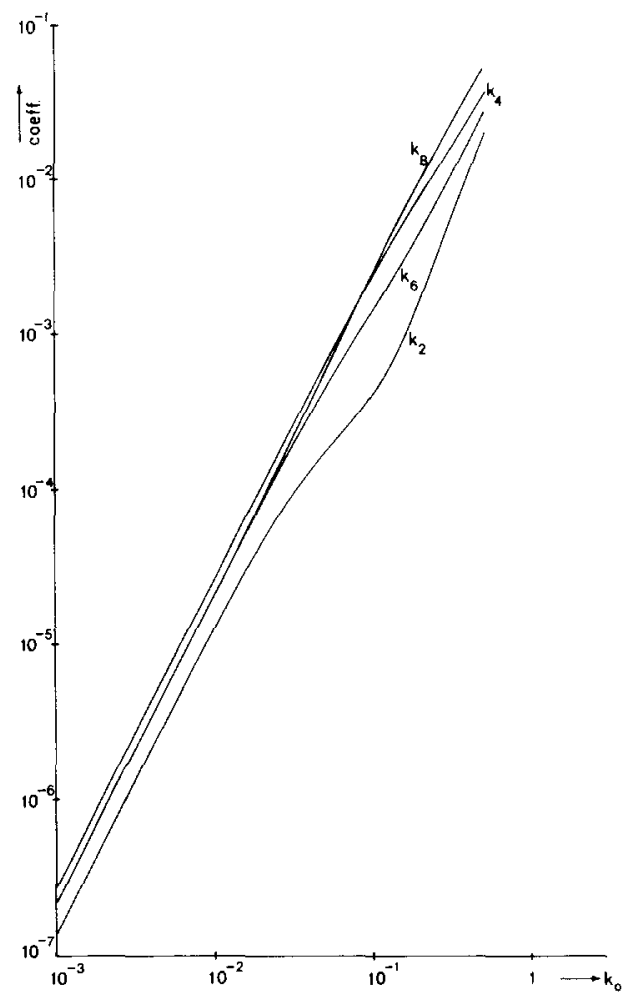

Fig. 4. The optimized values of $k_{2}, k_{4}, k_{6}$, and $k_{8}$ as a function of $k_{0} . k$ is an eighth-power function of $w$.

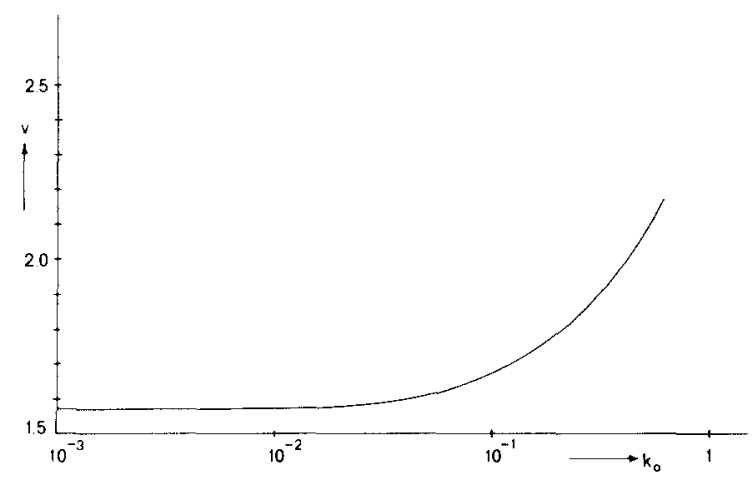

Fig. 5. The optimized value of $v$ as a function of $k_{0} . k$ is an eighth-power function of $w$.

$$
\begin{aligned}
- & {\left[f(0) f^{(10)}(0)+45 f^{(2)}(0) f^{(8)}(0)\right.} \\
+ & 210 f^{(4)}(0) f^{(6)}(0)+10 g^{(1)}(0) g^{(9)}(0) \\
+ & \left.120 g^{(3)}(0) g^{(7)}(0)+126 g^{(5)^{2}}(0)\right] / f^{3}(0)=0,
\end{aligned}
$$

where $f(u)$ and $g(u)$ are given by eqs. (15) and (16) together with (18) and (19) and where the exponent between brackets denotes the number of differentiations with respect to $u$ again.

The results of our computer calculations are shown in figs. 4 and 5 . In order to make convenient use of this type of profile, the results are given again in tabular form in table 2.

Table 2

\begin{tabular}{llllll}
$k=k_{0}+\frac{1}{2} k_{2} w^{2}+\frac{1}{24} k_{4} w^{4}+\frac{1}{720} k_{6} w^{6}+\frac{1}{40320} k_{8} w^{8}$ & & \\
\hline$k_{0}$ & $k_{2}$ & $k_{4}$ & $k_{6}$ & $k_{8}$ & $v$ \\
\hline 0.001 & $0.1344918 \mathrm{E}-6$ & $0.2685221 \mathrm{E}-6$ & $0.2176708 \mathrm{E}-6$ & $0.2138423 \mathrm{E}-6$ & 1.571336 \\
0.0015 & $0.3021345 \mathrm{E}-6$ & $0.6037009 \mathrm{E}-6$ & $0.4889647 \mathrm{E}-6$ & $0.4806909 \mathrm{E}-6$ & 1.571606 \\
0.002 & $0.5361709 \mathrm{E}-6$ & $0.1072431 \mathrm{E}-5$ & $0.8678578 \mathrm{E}-6$ & $0.8538265 \mathrm{E}-6$ & 1.571878 \\
0.003 & $0.1201322 \mathrm{E}-5$ & $0.2409479 \mathrm{E}-5$ & $0.1946312 \mathrm{E}-5$ & $0.1918264 \mathrm{E}-5$ & 1.572426 \\
0.005 & $0.3301244 \mathrm{E}-5$ & $0.6675282 \mathrm{E}-5$ & $0.5370816 \mathrm{E}-5$ & $0.5317301 \mathrm{E}-5$ & 1.573543 \\
0.007 & $0.6382663 \mathrm{E}-5$ & $0.1305242 \mathrm{E}-4$ & $0.1045596 \mathrm{E}-4$ & $0.1041026 \mathrm{E}-4$ & 1.574696 \\
0.01 & $0.1270177 \mathrm{E}-4$ & $0.2655204 \mathrm{E}-4$ & $0.2111624 \mathrm{E}-4$ & $0.2124108 \mathrm{E}-4$ & 1.576511 \\
0.015 & $0.2711895 \mathrm{E}-4$ & $0.5945364 \mathrm{E}-4$ & $0.4663559 \mathrm{E}-4$ & $0.4790848 \mathrm{E}-4$ & 1.579810 \\
0.02 & $0.4527998 \mathrm{E}-4$ & $0.1051987 \mathrm{E}-3$ & $0.8125040 \mathrm{E}-4$ & $0.8555060 \mathrm{E}-4$ & 1.583495 \\
0.03 & $0.8800870 \mathrm{E}-4$ & $0.2342309 \mathrm{E}-3$ & $0.1747778 \mathrm{E}-3$ & $0.1945888 \mathrm{E}-3$ & 1.592047 \\
0.05 & $0.1766461 \mathrm{E}-3$ & $0.6324751 \mathrm{E}-3$ & $0.4385705 \mathrm{E}-3$ & $0.5505032 \mathrm{E}-3$ & 1.613038 \\
0.07 & $0.2569693 \mathrm{E}-3$ & $0.1195166 \mathrm{E}-2$ & $0.7754077 \mathrm{E}-3$ & $0.1091081 \mathrm{E}-2$ & 1.636992 \\
0.1 & $0.3912534 \mathrm{E}-3$ & $0.2294400 \mathrm{E}-2$ & $0.1384272 \mathrm{E}-2$ & $0.2248266 \mathrm{E}-2$ & 1.674224 \\
0.15 & $0.8167910 \mathrm{E}-3$ & $0.4673770 \mathrm{E}-2$ & $0.2685508 \mathrm{E}-2$ & $0.5098914 \mathrm{E}-2$ & 1.733464 \\
0.2 & $0.1671438 \mathrm{E}-2$ & $0.7616743 \mathrm{E}-2$ & $0.4427084 \mathrm{E}-2$ & $0.9067361 \mathrm{E}-2$ & 1.787605 \\
0.3 & $0.4999499 \mathrm{E}-2$ & $0.1492337 \mathrm{E}-1$ & $0.9510110 \mathrm{E}-2$ & $0.1999371 \mathrm{E}-1$ & 1.885634 \\
0.5 & $0.1861874 \mathrm{E}-1$ & $0.3433195 \mathrm{E}-1$ & $0.2651986 \mathrm{E}-1$ & $0.5037162 \mathrm{E}-1$ & 2.069868 \\
\hline
\end{tabular}




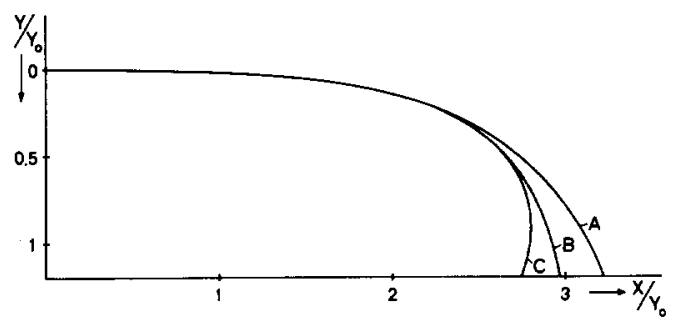

Fig. 6. The shape of the optimized profiles for three different cases. For the curves $\mathrm{A}, \mathrm{B}$, and $\mathrm{C}$ the $k$ function is respectively a constant, a fourth-power and an eighth-power function of $w$. The $k_{0}$ value is 0.02 .

\section{Comparison of different profiles; discussion}

In fig. 6 the shape is shown of the profiles for the three following cases: all three profiles have $k_{0}=0.02$; curve $\mathrm{A}$ is optimized for $k$ being a constant, curve $\mathrm{B}$ for $k$ being a fourth-power function in $w$ and curve $\mathrm{C}$ for $k$ being an eighth-power function in $w$. Curve $\mathrm{C}$ has a width that is 10 to $15 \%$ smaller than curve $\mathrm{A}$.

The field distribution at the surface of the profiles is very much different. Fig. 7 shows the field-strength distribution at the electrode surface for the corresponding profiles of fig. 6 . At the vertical axis the normalized difference has been plotted between the field strength at the position $x / y_{0}$ and the electrode centre. A great improvement of the field-strength uniformity is found. The question, however, is to which part the field-strength distribution in the space between the electrodes determines the discharge width. Therefore the field-strength distribution is also calculated at the midplane between the electrodes, where $v=0$. The results are plotted in fig. 7, curve $\mathrm{A}^{\prime}$. The distribution is essentially the same for all three cases. Experiments have to be carried out to determine the discharge width for the different profiles.

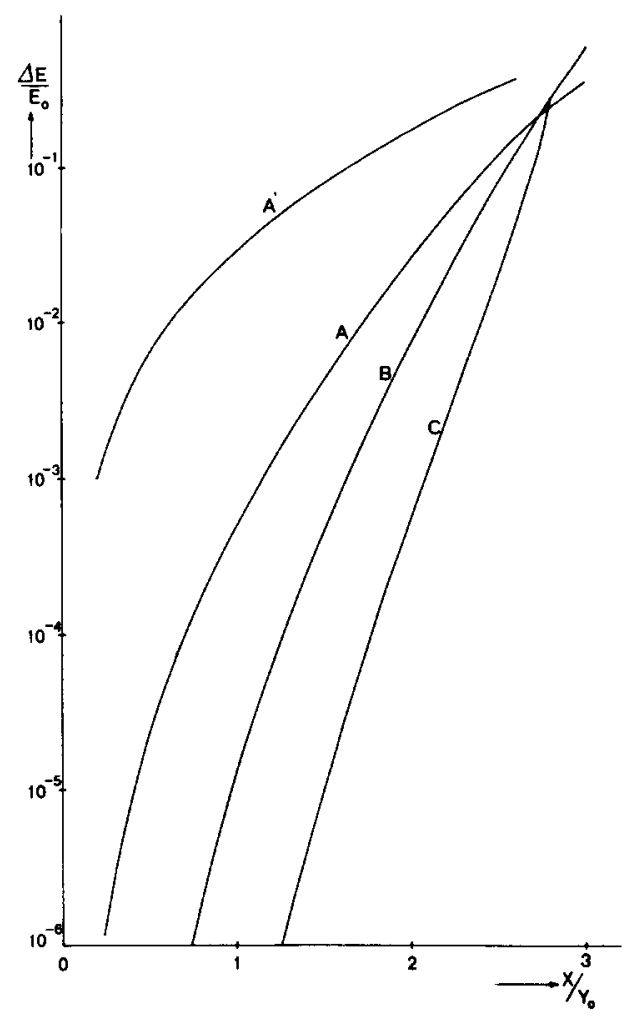

Fig. 7. The deviation of the electric field strength at the surface of the optimized electrodes from the central value as a function of the normalized position at the electrode surface. For the curves $\mathrm{A}, \mathrm{B}$, and $\mathrm{C}$ the $k$ function is respectively a constant, a fourth-power function and an eighth-power function of $w$. The $k_{0}$ value is 0.02 . The curve $\mathrm{A}^{\prime}$ gives the deviation of the electric-field strength at the midplane between the electrodes, also from its central value.

\section{References}

[1] W. Rogowski, Arch. Electrotech. 12 (1923) 1.

[2] T.Y. Chang, Rev. Scient. Instr. 44 (1973) 405.

[3] G.J. Ernst and A.G. Boer, Optics Comm. 27 (1978) 105. 